\title{
CDKN3 expression is negatively associated with pathological tumor stage and CDKN3 inhibition promotes cell survival in hepatocellular carcinoma
}

\author{
WEI DAI $^{1 *}$, HUILAI MIAO $^{1 *}$, SHUO FANG $^{2}$, TAO FANG $^{1}$, NIANPING CHEN $^{1}$ and MINGYI LI ${ }^{1}$ \\ Departments of ${ }^{1}$ Hepatobiliary Surgery and ${ }^{2}$ Endocrinology, Affiliated Hospital of Guangdong Medical University, \\ Zhanjiang, Guangdong 524001, P.R. China
}

Received July 11, 2015; Accepted May 31, 2016

DOI: $10.3892 / \mathrm{mmr} .2016 .5410$

\begin{abstract}
Aberrant expression of CDKN3 may be involved in carcinogenesis of liver cancer. The effect of CDKN3 on tumorigenesis and the molecular mechanisms involved have not been fully elucidated. Immunohistochemistry was performed to detect CDKN3 expression levels in tumor tissues. CDKN3 siRNA was used to knockdown CDKN3 in QGY7701 hepatocellular carcinoma (HCC) cells. Colony formation assay was used to measure the clonogenic capacity of the tumor cells. Cell viability was determined by MTT assay. Logistic regression was performed to analyze the association between CDKN3 expression level and the HCC clinical pathology index. The CDKN3 expression level was significantly decreased in HCC tumor tissues compared with normal liver tissue and liver cirrhosis tissue. Additionally, CDKN3 expression was negatively-associated with the pathological stage of the tumor. Inhibition of CKDN3 promoted the clonogenic capacity and chemotherapeutic tolerance in HCC tissues compared with controls. Knockdown of CDKN3 resulted in downregulation of p53 and p21 protein levels, whereas, AKT serine/threonine kinase 1 expression was upregulated. Thus, CDKN3 expression may reduce the survival of tumor cells and alter the sensitivity to therapeutic agents via the AKT/P53/P21 signaling pathway. Therefore, CDKN3 may be involved in tumor differentiation and self-renewal.
\end{abstract}

Correspondence to: Professor Mingyi Li or Dr Nianping Chen, Department of Hepatobiliary Surgery, Affiliated Hospital of Guangdong Medical University, 57 Renmingnan Road, Zhanjiang, Guangdong 524001, P.R. China

E-mail: limingyi63@163.com

E-mail:npc88@163.com

*Contributed equally

Key words: CDKN3, hepatocellular carcinoma, tumor suppressor

\section{Introduction}

Liver cancer is the second most common cause of cancer-associated mortality in China. The underlying molecular mechanisms that lead to the progression of liver cancer are unclear. Tumor-associated gene mutation, deletion and activation frequently lead to tumorigenesis. Previous studies have demonstrated that $\mathrm{p} 53, v$-myc avian myelocytomatosis viral oncogene homolog and hepatocyte growth factor are involved in the genesis of liver cancer (1-6). However, the complete process of cancer progression is far from understood.

Replicative immortality is an important hallmark of cancer. Cell cycle regulator dysfunction is an important event in carcinogenesis and progression. Cyclin-dependent kinase inhibitor 3 (CDKN3) is part of the dual-specificity protein phosphatase family. CDKN3 was identified as a cyclin-dependent kinase (CDK) inhibitor and interacts with, and dephosphorylates, CDK2 kinase, thus preventing its activation. CDKN3 was reported to be deleted, mutated, or overexpressed in several types of cancer (7-10). Previous studies have suggested that CDKN3 is expressed in hepatocellular carcinoma tissues and the overexpression of CDKN3 promotes cell proliferation in hepatoma cells $(11,12)$. CDKN3 may act as an oncogene in liver cancer, as CDKN3 is negative regulator of cell cycle via the inactivation of CDK2 (13-15). The present study investigated the CDKN3 protein expression pattern in liver cancer tissues and analyzed the association with pathological stage. Small interfering RNA (siRNA) was used to determine the importance of CDKN3 for tumor survival and cellular resistance to therapeutic agents.

\section{Materials and methods}

Cell culture. QGY7701 hepatocellular carcinoma (HCC) cells were used in the current study and were purchased from the Type Culture Collection of Chinese Academy of Sciences (Shanghai, China). QGY7701 cells were maintained in RPMI 1640 medium (Thermo Fisher Scientific, Inc., Waltham, MA, USA) supplemented with $10 \%$ fetal bovine serum (Thermo Fisher Scientific, Inc.) and $2 \mathrm{mM} \mathrm{L-glutamine.} \mathrm{Cells}$ were split every two days prior to reaching high confluence. Cells were cultured in a cell incubator with $5 \% \mathrm{CO}_{2}$ at $37^{\circ} \mathrm{C}$. 
Table I. Characteristics of age, gender and HBV infection in hepatocellular carcinoma, normal liver and liver cirrhosis group.

\begin{tabular}{lcccccr}
\hline Characteristic & $\begin{array}{c}\text { Cancer/normal } \\
(\mathrm{n}=70 / 10)\end{array}$ & $\begin{array}{c}\text { Odds } \\
\text { ratio }\end{array}$ & $95 \% \mathrm{CI}$ & $\begin{array}{c}\text { Cancer/cirrhosis } \\
(\mathrm{n}=70 / 6)\end{array}$ & $\begin{array}{c}\text { Odds } \\
\text { ratio }\end{array}$ & $95 \% \mathrm{CI}$ \\
\hline Age & & & & & & \\
$<50$ & $26 / 4$ & 1.00 & & $26 / 2$ & 1.00 & \\
$>=50$ & $44 / 6$ & 1.13 & $0.29-4.370$ & $44 / 4$ & 0.85 & $0.14-4.940$ \\
Gender & & & & & \\
Male & $63 / 7$ & 1.00 & & $63 / 5$ & 1.00 & $0.05-5.460$ \\
Female & $7 / 3$ & 0.26 & $0.05-1.240$ & $7 / 1$ & & \\
HBV & & & & $61 / 5$ & 1.00 & \\
No & $61 / 2$ & 1.00 & & $9 / 1$ & 1.36 & $0.14-12.97$ \\
Yes & $9 / 8$ & 27.11 & $4.95-148.440^{\mathrm{a}}$ & & & \\
\hline
\end{tabular}

a, $\mathrm{P}=0.041$ vs. the normal group. HBV, hepatitis B virus; $\mathrm{CI}$, confidence intervals.

HCC tissue sample and immunohistochemistry (IHC). A total of 70 samples of HCC tissues, 6 samples of liver cirrhosis tissues and 10 samples of normal liver tissues were separately obtained following surgical resection. The present study was approved by the ethics committee of the Affiliated Hospital of Guangdong Medical University (Zhanjiang, China) and written informed consent was obtained. Patients with $\mathrm{HCC}$ had not received radiation/chemotherapy prior to surgery. The characteristics of each group are presented in Table I. Cancer tissues were collected following surgical dissection and immediately fixed in $10 \%$ formalin overnight at room temperature. Subsequently, the fixed tissues were dehydrated and embedded in paraffin. In order to visualize the target proteins, the samples were deparaffinized in xylene and then rehydrated via a graded alcohol series. The tissue sections were then washed with PBS and heated twice in a microwave oven for $5 \mathrm{~min}$ in citrate buffer ( $\mathrm{pH} \mathrm{6)}$. Sections were then incubated with CDKN3-specific antibody (Abnova Corporation, Taipei, Taiwan; cat. no. orb100671) at a dilution of 1:200.Horseradish peroxidase-labeled goat anti-rabbit secondary antibody (Beyotime Institute of Biotechnology, Haimen, China; cat. no. A0208) was used at a dilution of 1:500. Following hematoxylin staining, the sections were dried and sealed with a cover slip. The protein signal was observed under a fluorescence microscope (DM2000; Leica Microsystems GmbH, Wetzlar, Germany) and evaluated by a pathologist. The CDKN3-positive cell number and staining degree of each section was assessed by the same pathologist (Dr Shuo Fang, Guangdong Medical University, Zhanjiang, China) and samples were scored from the highest to the lowest scores (5-1 scores). The pathological stage was evaluated by Dr Wei Dai (Guangdong Medical University).

RNA interference. A CDKN3-specific siRNA knockdown kit was purchased from Shanghai GenePharma Co., Ltd. (Shanghai, China). Lipofectamine RNAiMAX (Thermo Fisher Scientific, Inc.) was used to transfect 20 pmol siRNA to each well of a $24-$ well plate with cell density of $3 \times 10^{5}$. This experiment was performed according the manufacturer's protocol.

Cell viability assay. Cells with CDKN3 knockdown were seeded in 96-well plates at a density of 5,000 cells/well. After
$12 \mathrm{~h}$ culture, cells were treated with various doses of cisplatin (Hansoh Pharmaceutical Group Co., Ltd., Linyungang, China) and cell viability was measured. Briefly, following cisplatin treatment, $10 \mu 1$ 3-(4,5-dimethylthiazol-2-yl)-2,5-diphenyltetrazolium bromide (MTT) solution $(5 \mathrm{mg} / \mathrm{ml})$ was added to each well. The cells were incubated at $37^{\circ} \mathrm{C}$ for $2 \mathrm{~h}$. The medium was removed and $100 \mu \mathrm{l}$ dimethyl sulfoxide was added to dissolve the formazan crystals. The absorbance at $570 \mathrm{~nm}$ was determined using a spectrophotometer when the crystals were completely dissolved.

Clonogenic survival assay. Cells were diluted with RPMI medium and seed at 500 cells/well in a 6-well plate. Cells were cultured for 7-10 days, then the medium was removed and cells were stained with $0.1 \%$ crystal violet for $5 \mathrm{~min}$. Next, the cells were air-dried at room temperature and the plates were imaged. The number of colonies in each well was manually counted.

Western blotting. Cells were collected and total protein was extracted with radioimmunoprecipitation assay buffer supplemented with $1 \mathrm{mM}$ phenylmethylsulfonyl fluoride. Protein $(20 \mu \mathrm{g})$ was loaded onto gels and separated by electrophoresis on a $10 \%$ SDS-PAGE gel, the protein was then transferred to a polyvinylidene difluoride membrane $(250 \mathrm{~mA}, 2 \mathrm{~h})$. The membranes containing protein blots were incubated in blocking buffer (5\% non-fat milk) for $1 \mathrm{~h}$ at room temperature, prior to the addition of primary antibodies. Rabbit primary antibodies against AKT serine/threonine kinase (AKT; cat. no. 4685), p53 (cat. no. 2527), p21 (cat. no. 2947; Cell Signaling Technology, Inc., Danvers, MA, USA) and CDKN3 (Abnova Corporation) were used at a dilution of 1:500 and incubated overnight at $4^{\circ} \mathrm{C}$. The horseradish peroxidase-conjugated goat anti-rabbit secondary antibody (EarthOx Life Sciences, Millbrae, CA, USA; cat. no. E030120-01) was used in dilution of 1:5,000 and incubated at room temperature for 1-2 h. Tris-buffered saline supplemented with $0.5 \%$ Triton X-100 was used as the washing buffer. An ECL western blotting substrate (Pierce; Thermo Fisher Scientific, Inc.) was used to detect the bands and this was visualized with $\mathrm{X}$-ray film. 
A

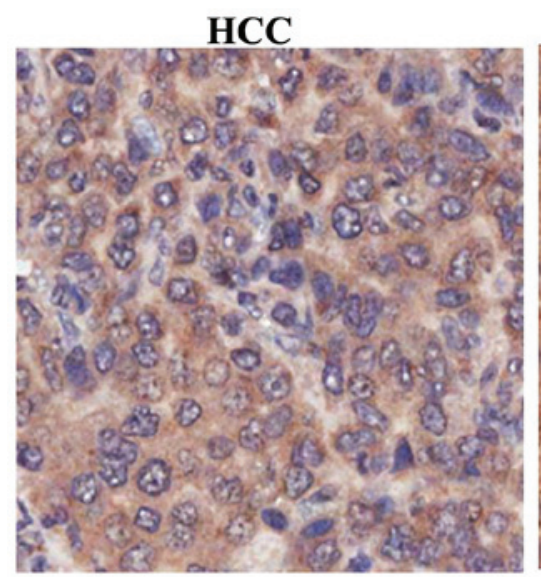

Liver cirrhosis

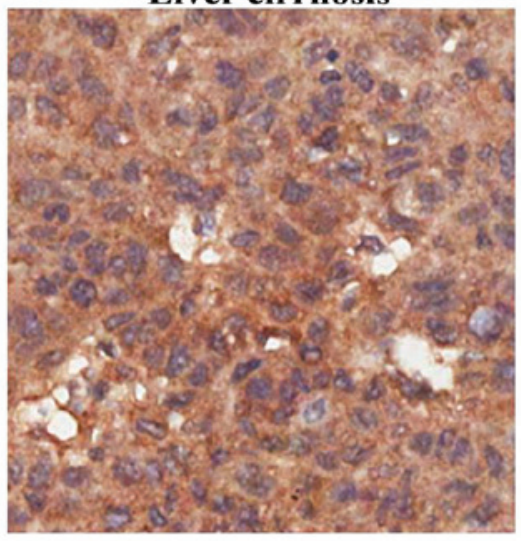

Normal liver

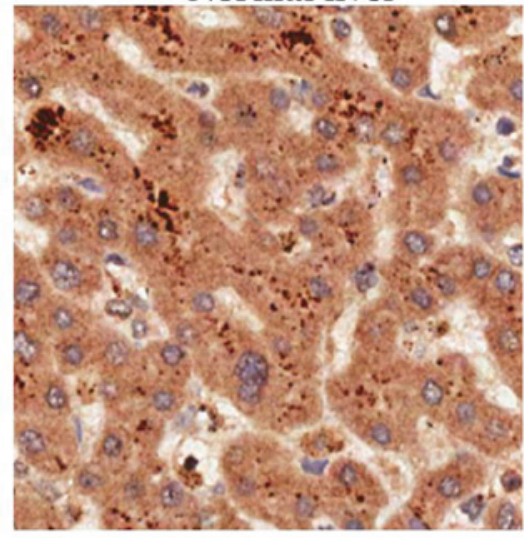

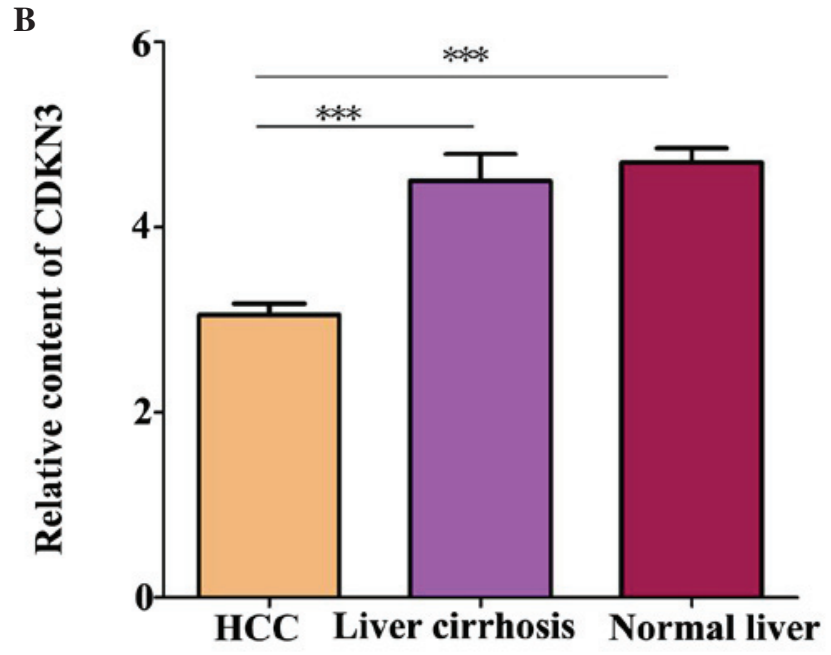

Figure 1. CDKN3 expression is downregulated in HCC tissues. (A) CDKN3 protein in normal liver, liver cirrhosis and HCC tissues was detected using immunohistochemistry. (B) CDKN3 expression content in the different tissues was evaluated. ${ }^{* * *} \mathrm{P}<0.001, \mathrm{HCC}$ vs. liver cirrhosis; ${ }^{* * *} \mathrm{P}<0.001, \mathrm{HCC}$ vs. normal liver. CDKN3, cyclin-dependent kinase inhibitor 3; HCC, hepatocellular carcinoma.

Statistical analysis. Data were collected, analyzed and presented as the mean \pm standard deviation. One-way analysis of variance and Tukey's honest significant difference test was performed to compare differences among multiple groups. Odds ratio and $95 \%$ confidence interval were applied to verify the characteristic differences between each group. Logistic regression analysis was used to examine the association between CDKN3 expression and the tumor clinical pathology index. Statistical analysis was conducted on Prism 5.0 software (GraphPad Software, Inc., La Jolla, CA, USA) and $\mathrm{P}<0.05$ was considered to indicate a statistically significant difference.

\section{Results}

CDKN3 expression is downregulated in HCC tissues. CDKN3 protein level in tissues was detected by IHC. A total of $70 \mathrm{HCC}$ tissue, 10 liver tissue and 6 liver cirrhosis tissue samples were examined. The characteristics of the samples in each group are presented in Table I. Statistical analysis identified no significant difference in age and gender among the groups. Notably, the hepatitis B virus (HBV) infection ratio was significantly higher in $\mathrm{HCC}$ tissues compared with normal liver tissues $(\mathrm{P}=0.041)$. Additionally, $\mathrm{CDKN} 3$ expression levels were higher in normal and liver cirrhosis tissues compared with HCC tissues (Fig. 1; P=0.00034).

CDKN3 expression is associated with tumor stage. Tumor pathological stage was evaluated according to the cell nuclear morphology and the karyoplasmic ratio. This is a vital factor for cancer prognosis. A tumor at a high stage is associated with poor prognosis. The HCC tumor stage was evaluated and the association between CDKN3 expression and tumor stages was analyzed (Fig. 2A). It was determined that CDKN3 is negatively associated with tumor stage (Fig. 2B; $\mathrm{P}<0.00026$ ). Thus, CDKN3 expression levels were downregulated in high stage cancer with immature tumor cells.

Depletion of CDKN3 increases cell survival and cisplatin tolerance. To determinate the function of CDKN3 in QGY7701 cells, CDKN3 was knocked-down by transfection with CDKN3-specific siRNA. The knockdown of CDKN3 enhanced the colony formation capacity of the cells at $12 \mathrm{~h}$. Larger tumor cell colonies formed following CDKN3 knockdown compared 
A

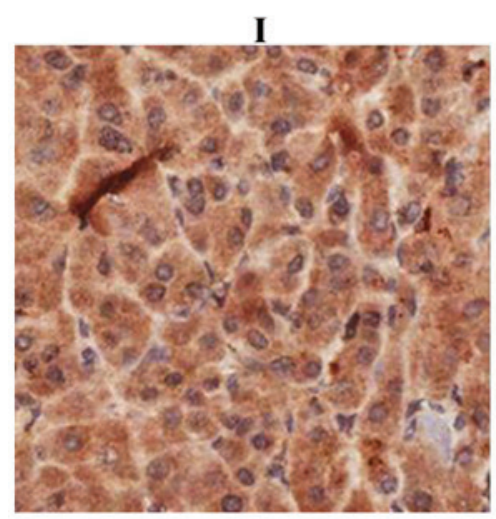

III

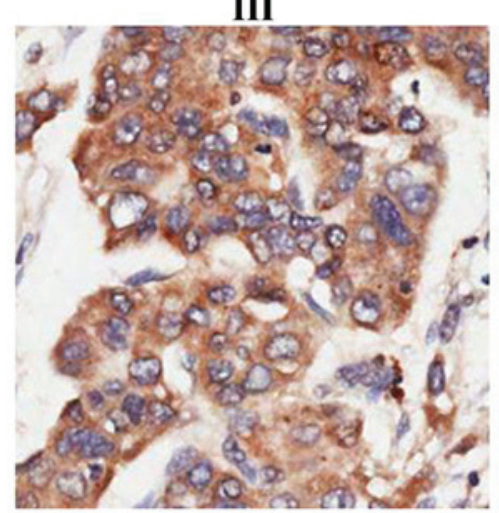

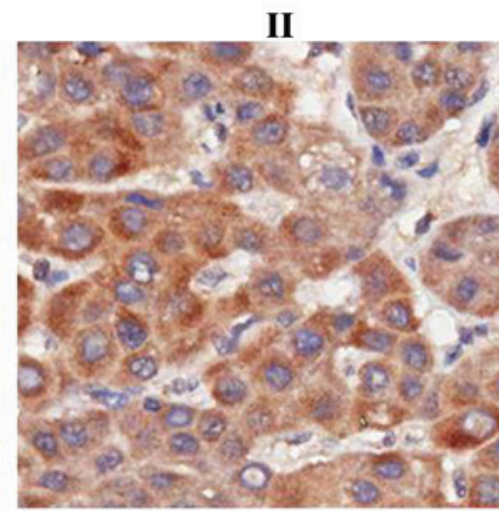

IV

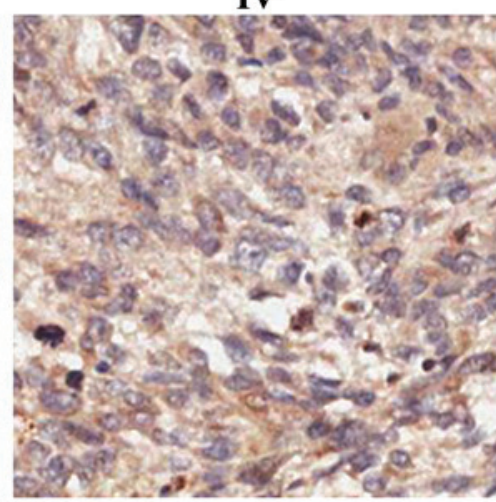

B

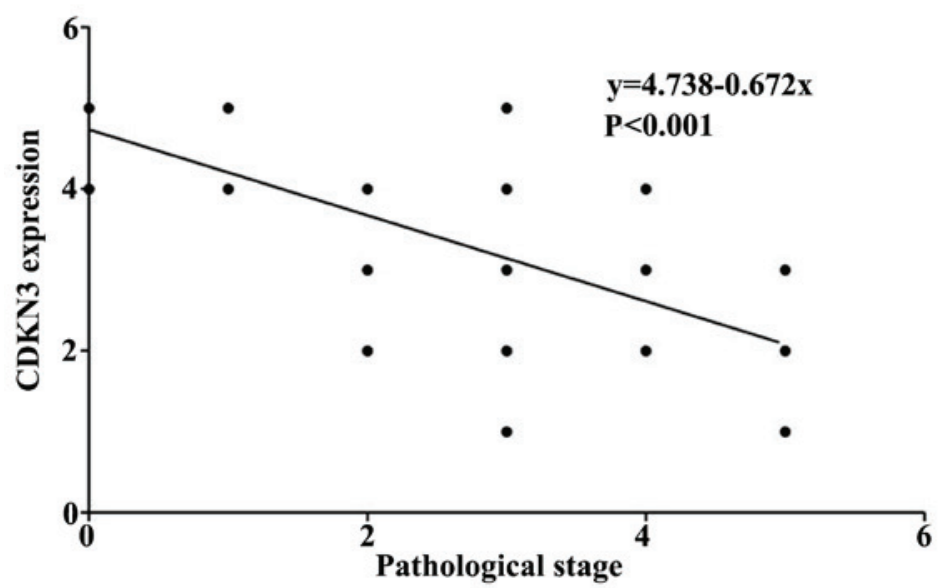

Figure 2. CDKN3 expression is correlated with tumor stage. (A) CDKN3 expression at hepatocellular carcinoma tumor stages I-IV were determined using immunohistochemistry. (B) The association between CDKN3 expression and tumor stage was determined using logistic regression analysis. CDKN3, cyclin-dependent kinase inhibitor 3.

with the control group, in addition, the number of colonies also increased compared with the control group (Fig. 3A and B). Cisplatin is frequently used for the treatment of cancer. Thus, QGY7701 cells were treated with cisplatin following CDKN3 knockdown and cell viability was measured. QGY7701-KD cells exhibited a higher tolerance to cisplatin compared with the control group ( $\mathrm{P}=0.024$; Fig. $3 \mathrm{C}$ and $\mathrm{D})$.

Knockdown of CDKN3 activates the AKT/p53/p21 signaling pathway. The AKT/phosphatidylinositol-4,5-bisphosphate 3-kinase (PI3K) pathway was investigated for possible alterations in signaling. It was demonstrated that AKT expression levels were upregulated and the downstream proteins, including p53 and p21 were decreased by CDKN3 siRNA compared with scramble controls (Fig. 3E). These results suggested that low expression levels of CDKN3 promote the activation of the AKT/PI3K signaling pathway and inhibit p53 and p21, which contributes to the survival of tumor cells and increases their tolerance to cisplatin cytotoxicity.

\section{Discussion}

Liver cancer is the second most common cause of cancer-associated mortality and its incidence in China is increasing. A previous epidemiological study demonstrated that HBV-infection was the primary cause of liver cancer in 
A

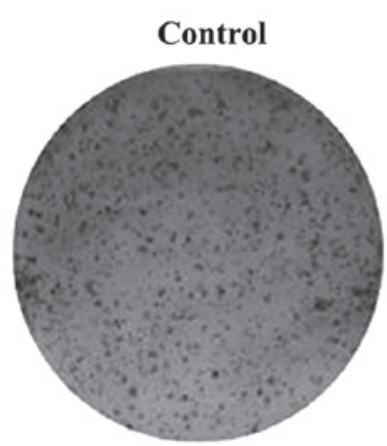

Cisplatin

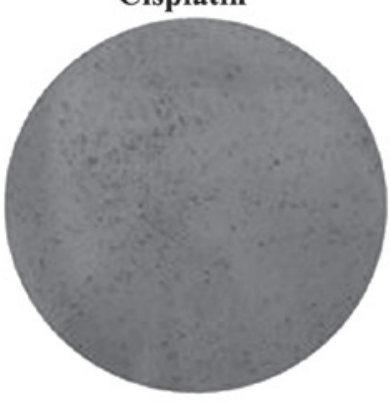

C

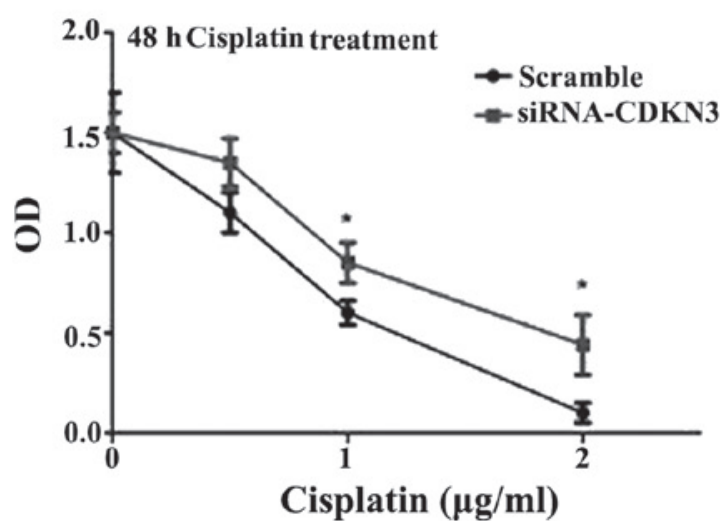

B

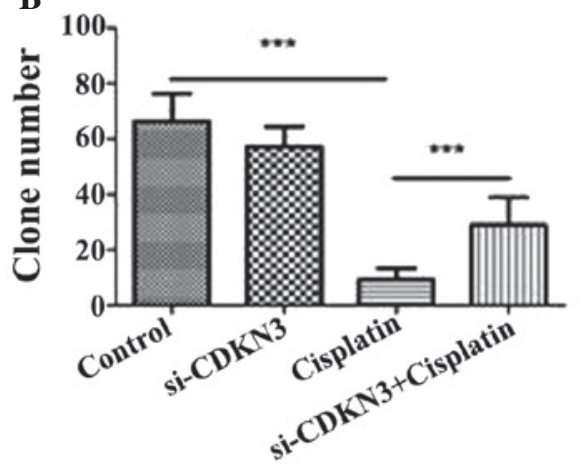

D

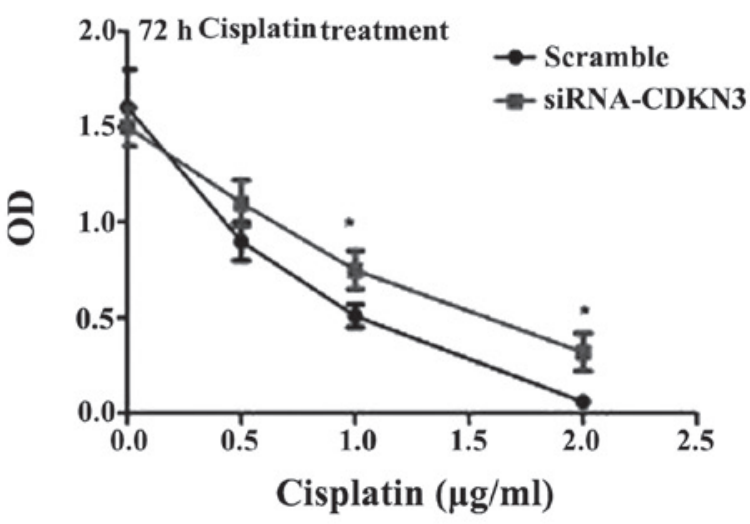

E Scramble si-CDKN3

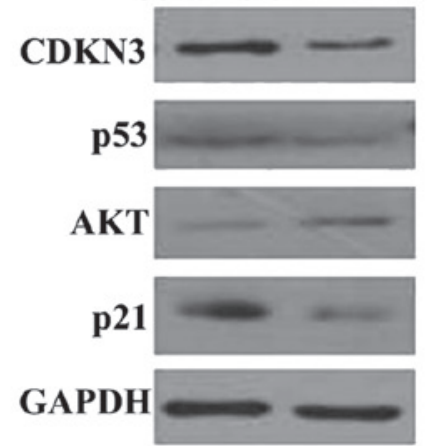

Figure 3. Depletion of CDKN3 increased cell survival and cisplatin tolerance by activation of the AKT/p53/p21 pathway. (A) Tumor clone formation assay was performed to test clone formation capacity of different tumor cells. (B) Clone formation assay data. ${ }^{* * *} \mathrm{P}<0.001$, comparison indicated by brackets. Cell viability of cells was assessed by 3-(4,5-dimethylthiazol-2-yl)-2,5-diphenyltetrazolium bromide assay following (C) 48 h and (D) 72 h cisplatin treatment. ${ }^{*} \mathrm{P}<0.05$ vs. the scramble control. (E) Western blotting was used to detect the levels of the AKT/p53/p21 signaling pathway proteins. siRNA, small interfering RNA; CDKN3, cyclin-dependent kinase inhibitor 3; AKT, AKT serine/threonine kinase 1; OD, optical density.

China (16). Further investigation of liver carcinogenesis in Chinese patients is vital for the efficient treatment of liver cancer.
Deregulation of the cell cycle results in uncontrolled proliferation and apoptosis-resistant tumor cells. Abnormal changes in cell cycle-associated proteins associated with tumorigenesis. 
CDKN3 dephosphorylates threonine-161 of CDK1 during mitosis, which is essential for normal G1/S transition. A previous study identified that CDKN3 is downregulated in brain tumors (15). However, previous studies have also demonstrated high expression of CDKN3 in various types of tumor $(9,17,18)$. Thus, the importance of CDKN3 in carcinogenesis has not been fully elucidated. A previous study demonstrated overexpression of CDKN3 in liver cancer tissues, however, whether CDKN3 expression is clinically important remains uncertain and the detailed molecular mechanisms of CDKN3 in the development and progression of liver cancer remain unclear.

The present study analyzed CDKN3 expression in HCC tissues. The CDKN3 expression levels between normal and liver cancer tissues were compared. CDKN3 expression was measured in 10 normal liver tissues, CDKN3 exhibited higher expression in normal liver tissues compared with tumor tissues. The HBV infection ratio was higher in patients with HCC tissues compared with controls, and CDKN3 was consistently expressed at a higher level in normal liver tissues compared with HCC tissues. The results of the current study suggest that CDKN3 is a highly expressed in normal liver tissues. Thus, downregulation of CDKN3 may enhance the progression of liver cancer. It was also demonstrated, that CDKN3 expression was associated with the differentiation stage of tumors, therefore, $\mathrm{CDKN} 3$ may affect tumor proliferation, metamorphosis and invasion.

To determine the function of CDKN3 in the tumor cells, siRNA interference was performed to deplete CDKN3 levels in liver cancer cells. To investigate the impact of CDKN3 expression on the cell viability of tumor cells, MTT assay was performed to determine the changes in cell viability. Inhibition of CDKN3 did not have an effect on the cell viability; however, it did affect the reactivity to cisplatin. It is possible that CDKN3 expression promotes the transduction of apoptotic signals, which is important for the elimination of unhealthy cells and prevention of malignant transformation of cells. Colonic potential was determined by colony formation assay and the knockdown of CDKN3 promoted the formation of larger cell colonies. The AKT/p53/p21 signaling pathway is vital for the survival of tumor cells, these proteins were quantified using western blot analysis. It was demonstrated that depletion of CDKN3 may lead to the activation of the AKT/p53/p21 signaling pathway (19). Thus, high expression levels of CDKN3 may be beneficial for normal cells. Low expression of CDKN3 may be a marker for poor liver cancer prognosis.

The results of the current study contrast with previous studies $(12,14)$. This may because previous studies detected CDKN3 mRNA expression levels as opposed to protein expression levels. Additionally, normal liver tissues were not tested in the previous studies in order to confirm the changes of CDKN3 expression. In the present study, CDKN3 expression was associated with the pathological tumor stage. Therefore, the tumor sample collection (tumor stage distribution and number of cases) is important for correct conclusions to be reached. The current study collected samples from 70 HCC cases with integral case information, including tumor stage, age, gender and $\mathrm{HBV}$ infection status. CDKN3 protein was quantified using IHC. Characteristics, including age, gender and HBV infection status of each group were examined using odds ratio and $95 \%$ confidence interval. It was observed that there was no difference in age and gender between the cancer and normal groups, suggesting this does not result in the CDKN3 alteration. However, the HBV infection ratio was higher in the cancer group compared with normal people, however, further research is required to elucidate the association between CDKN3 expression and HBV infection. In conclusion, CDKN3 may act as a tumor suppressor in liver tissues by modulation of the cell survival signal transduction, monitoring carcinogenesis and elimination of abnormal cells.

\section{References}

1. Shiraha H, Yamamoto K and Namba M: Human hepatocyte carcinogenesis (review). Int J Oncol 42: 1133-1138, 2013.

2. Zimonjic DB and Popescu NC: Role of DLC1 tumor suppressor gene and MYC oncogene in pathogenesis of human hepatocellular carcinoma: Potential prospects for combined targeted therapeutics (review). Int J Oncol 41: 393-406, 2012.

3. Kirstein MM and Vogel A: The pathogenesis of hepatocellular carcinoma. Dig Dis 32: 545-553, 2014.

4. Meng X, Franklin DA, Dong J and Zhang Y: MDM2-p53 pathway in hepatocellular carcinoma. Cancer Res 74: 7161-7167, 2014.

5. Kawaguchi M and Kataoka H: Mechanisms of hepatocyte growth factor activation in cancer tissues. Cancers (Basel) 6: 1890-1904, 2014.

6. Xie B and Dong JH: HGF/c-Met and metastasis of hepatocellular carcinoma. Zhonghua Gan Zang Bing Za Zhi 13: 396-398, 2005 (In Chinese).

7. Espinosa AM, Alfaro A, Roman-Basaure E, Guardado-Estrada M, Palma Í, Serralde C, Medina I, Juárez E, Bermúdez M, Márquez $\mathrm{E}$, et al: Mitosis is a source of potential markers for screening and survival and therapeutic targets in cervical cancer. PLoS One 8: e55975, 2013.

8. Lai MW, Chen TC, Pang ST and Yeh CT: Overexpression of cyclin-dependent kinase-associated protein phosphatase enhances cell proliferation in renal cancer cells. Urol Oncol 30: 871-878, 2012.

9. Li T, Xue H, Guo Y and Guo K: CDKN3 is an independent prognostic factor and promotes ovarian carcinoma cell proliferation in ovarian cancer. Oncol Rep 31: 1825-1831, 2014.

10. Taylor KJ, Sims AH, Liang L, Faratian D, Muir M, Walker G, Kuske B, Dixon JM, Cameron DA, Harrison DJ and Langdon SP: Dynamic changes in gene expression in vivo predict prognosis of tamoxifen-treated patients with breast cancer. Breast Cancer Res 12: R39, 2010

11. Drozdov I, Bornschein J, Wex T, Valeyev NV, Tsoka S and Malfertheiner P: Functional and topological properties in hepatocellular carcinoma transcriptome. PLoS One 7: e35510, 2012.

12. Xing C, Xie H, Zhou L, Zhou W, Zhang W, Ding S, Wei B, Yu X, Su R and Zheng S: Cyclin-dependent kinase inhibitor 3 is overexpressed in hepatocellular carcinoma and promotes tumor cell proliferation. Biochem Biophys Res Commun 420: 29-35, 2012.

13. Li H, Jiang X, Yu Y, Huang W, Xing H, Agar NY, Yang HW, Yang B, Carroll RS and Johnson MD: KAP regulates ROCK2 and Cdk2 in an RNA-activated glioblastoma invasion pathway. Oncogene 34: 1432-1441, 2015.

14. Yeh CT, Lu SC, Chen TC, Peng CY and Liaw YF: Aberrant transcripts of the cyclin-dependent kinase-associated protein phosphatase in hepatocellular carcinoma. Cancer Res 60: 4697-4700, 2000.

15. Nalepa G, Barnholtz-Sloan J,Enzor R, Dey D, He Y, Gehlhausen JR, Lehmann AS, Park SJ, Yang Y, Yang X, et al: The tumor suppressor CDKN3 controls mitosis. J Cell Biol 201: 997-1012, 2013.

16. Hou J, Liu Z and Gu F: Epidemiology and prevention of hepatitis B virus infection. Int J Med Sci 2: 50-57, 2005.

17. Yu Y, Jiang X, Schoch BS, Carroll RS, Black PM and Johnson MD: Aberrant splicing of cyclin-dependent kinase-associated protein phosphatase KAP increases proliferation and migration in glioblastoma. Cancer Res 67: 130-138, 2007.

18. Lee SW, Reimer CL, Fang L, Iruela-Arispe ML and Aaronson SA: Overexpression of kinase-associated phosphatase (KAP) in breast and prostate cancer and inhibition of the transformed phenotype by antisense KAP expression. Mol Cell Biol 20: 1723-1732, 2000.

19. McCubrey JA, Steelman LS, Franklin RA, Abrams SL, Chappell WH, Wong EW, Lehmann BD, Terrian DM, Basecke J, Stivala F, et al: Targeting the RAF/MEK/ERK, PI3 K/AKT and p53 pathways in hematopoietic drug resistance. Adv Enzyme Regul 47: 64-103, 2007. 